Ergod. Th. \& Dynam. Sys. (1986), 6, 193-203

Printed in Great Britain

\title{
Vector fields with transverse foliations, II
}

\author{
SUE GOODMAN \\ Department of Mathematics, University of North Carolina, Chapel Hill, \\ NC 27514, USA
}

(Received 15 September 1984 and revised 9 April 1985)

\begin{abstract}
When does a non-singular flow on a 3-manifold have a 2-dimensional foliation everywhere transverse to it? A complete answer is given for a large class of flows, those with 1-dimensional hyperbolic chain recurrent set. We find a simple necessary and sufficient condition on the linking of periodic orbits of the flow.
\end{abstract}

\section{Introduction}

Given a non-singular vector field on a 3-dimensional manifold, is there a 2 dimensional foliation everywhere transverse to it? In general, no. For example, if one considers the unit tangent vector field to a circle bundle over a surface, Wood [W] has shown there is a transverse foliation if and only if the Euler number of the bundle is less than minus the Euler characteristic of the surface (for surfaces of genus 1 or more). Similar results have been obtained for Seifert bundles [E-H-N]. In another direction, Fried [Fri] and Schwartzman [Sch] have given conditions for a flow to have a cross-section, hence a transverse foliation.

D. Asimov and the author ([A-G], $[\mathbf{G}]$ ) have found a simple linking property between periodic orbits that is necessary for any flow to have a transverse foliation. Any null-homotopic periodic orbit must link, that is any disk it bounds must intersect, a periodic orbit. For flows with a 1-dimensional hyperbolic chain recurrent set, a slightly stronger condition is necessary. Any null-homotopic periodic orbit must link an attracting or repelling periodic orbit.

However neither version of the linking property is, in general, sufficient for a non-singular flow to have a transverse foliation. For example, the unit tangent vector field to an $\boldsymbol{S}^{\mathbf{1}}$-bundle of sufficiently large Euler number over a surface of genus 2 has no transverse foliation but satisfies either linking property vacuously. But for some classes of flows this second version of the linking propery is sufficient. In [G], the author showed this for non-singular Morse-Smale flows. Here we show that it is also sufficient for Smale flows, or more generally, flows with a hyperbolic 1dimensional chain recurrent set. Unlike Morse-Smale flows, which occur only on certain 3-manifolds [M], Smale flows are abundant. In fact, Zeeman [Z] has claimed they are dense in the $C^{0}$-topology of non-singular vector fields. A proof appears in an unpublished paper of Oliveira [O].

In $\S 1$ of this paper, we give some preliminary background. In $\S 2$, we prove the necessity of the hyperbolic version of the linking property. In $\S 3$, the main body 
of the paper, we show sufficiency. Some of the foliations we construct are only $C^{1}$-smooth. However it was shown in [G] that this is the best possible for some flows; that is, there are flows which have a transverse foliation but no $C^{2}$-smooth one.

The extension of this work to Smale flows was suggested by Dennis Sullivan and the author would like to express her appreciation.

\section{Preliminaries and statement of main theorem}

We consider here non-singular flows which have a 1-dimensional hyperbolic chain recurrent set. All flows will be on closed oriented 3-manifolds. If we were also to require that for every $x, y$ in the chain recurrent set, the stable manifold of $x$ and the unstable manifold of $y$ meet transversally, the flow would be a Smale flow (see [Fra1] for a general discussion of Smale flows). This additional assumption makes the flow structurally stable, but we shall not require it in this paper.

Hyperbolicity of the chain recurrent set $R$ of a flow $\varphi$ implies ([Sm]) that $R$ can be decomposed into a finite disjoint union of compact invariant sets $\Lambda_{0}, \ldots, \Lambda_{n}$, called basic sets, each having an orbit dense in that basic set. This decomposition gives rise to a filtration of the manifold $M$; that is, a collection of submanifolds $M_{0} \subseteq M_{1} \subseteq \cdots \subseteq M_{n}=M$ such that:

(a) $\varphi_{t}\left(M_{i}\right) \subseteq$ interior $M_{i}$; and

(b) $\Lambda_{i}=\bigcap_{t} \varphi_{t}\left(M_{i}-M_{i-1}\right)$.

The filtration is obtained by taking $M_{i}=g^{-1}\left(-\infty, r_{i}\right]$ where $g: M \rightarrow \mathbb{R}$ is a Lyapunov function for $\varphi$ and the $r_{i}$ 's are regular values of $g$ separating the basic sets. We may assume that the basic sets are all at different critical levels. We may also assume that each $M_{i}-M_{i-1}$ is connected (although we may lose the direct association with a Lyapunov function).

As in [G], a simple linking property between periodic orbits of the flow is both necessary and sufficient for the flow to have a 2-dimensional foliation everywhere transverse to it.

Linking property. Any periodic orbit $\sigma$ which is null-homotopic in $M$ has the property that the interior of any disk $D$ bounded by $\sigma$ meets an attracting or repelling periodic orbit.

Our main theorem is:

THEOREM 3.1. A non-singular flow $\varphi$ with hyperbolic 1-dimensional chain recurrent set on a closed oriented 3-manifold $M$ has a 2-dimensional foliation everywhere transverse to it if and only if $\varphi$ satisfies the linking property.

We will prove the necessity of the linking property in $\S 2$ and the sufficiency in $\S 3$.

\section{Necessity of the linking property}

The linking property used in [A-G], [G] was stated as follows: any null-homotopic periodic orbit must have the property that the interior of any disk it bounds must meet a periodic orbit. We proved that this linking property was necessary for any non-singular flow to have a transverse foliation. For the purposes of this paper we need the slightly stronger version stated in $\S 1$ which we now prove is necessary for flows with hyperbolic chain recurrent set. 
LEMMA 2.1. Any flow $\varphi$ with 1-dimensional hyperbolic chain recurrent set which is transverse to a Reeb component (a foliation of $D^{2} \times S^{1}$ ) must have an attracting or repelling periodic orbit contained in the interior of the Reeb component and homotopic to the generator of $\pi_{1}\left(D^{2} \times S^{1}\right)$.

Proof. Assume the flow is oriented in on the boundary of the Reeb component. Novikov ([No]) has shown that there is a cross-sectional disk $D$ of $D^{2} \times S^{1}$, transverse to the flow and with a first-return map $r: D \rightarrow$ int $D$. Basically one takes a disk in a planar leaf of the Reeeb component whose boundary is very near the boundary of the Reeb component and extends this disk to the boundary, staying transverse to $\varphi$. The existence of the first-return map is clear since the flow is transverse to the Reeb foliation.

Any fixed point of the map $r$ corresponds to a periodic orbit of $\varphi$, so it is hyperbolic. Hence the fixed points are isolated. An index argument on the disk (whose Euler characteric is 1) shows that some fixed point is a sink or source. It then corresponds to an attracting or repelling periodic orbit of $\varphi$, homotopic to the generator of $\pi_{1}\left(D^{2} \times S^{1}\right)$.

Proposition 2.2. Any flow $\varphi$ with hyperbolic chain recurrent set which has a transverse foliation must satisfy the linking property.

The argument is exactly as in [A-G], [G]. If the flow $\varphi$ has a transverse foliation $F$ and if $\sigma$ is a null-homotopic periodic orbit bounding an immersed disk $D$, then $D$ may be perturbed slightly to $D^{\prime}$ in general position with respect to $F$. By Novikov ([No]), since $\sigma$ is a null-homotopic closed curve transverse to $F, D^{\prime}$ must contain a meridian circle of a Reeb component. By the above lemma, $D^{\prime}$ intersects an attractor or repeller of the flow $\varphi$ with non-zero algebraic intersection. Hence $D$ also meets this orbit, satisfying the linking property.

COROLlary 2.3. If the flow $\varphi$ satisfies the linking property then no periodic orbit in a basic set $\Lambda_{i}$ is null-homotopic in $M_{i}-M_{i-1}$.

Proof. If the periodic orbit is an attractor or repeller then $\overline{M_{i}-M_{i-1}}$ is homeomorphic to a solid torus $D^{2} \times S^{1}$ with the orbit homotopic to the generator of $\pi_{1}\left(D^{2} \times S^{1}\right)$.

If the periodic orbit is in a basic set $\Lambda_{i}$, not an attractor or repeller, and bounds a disk in $M_{i}-M_{i-1}$, then that disk clearly cannot meet an attractor or repeller, since they compose other basic sets. This contradicts the linking property.

It is in this last form that we shall use the linking property to show sufficiency.

\section{Sufficiency of the linking property}

We assume now that $\varphi$ is a non-singular flow with hyperbolic 1-dimensional chain recurrent set, satisfying the linking property hence the property of corollary 2.3. The flow restricted to any basic set is topologically equivalent to a subshift of finite type with an irreducible matrix ([B]). We shall construct a foliation transverse to op with a finite number of compact leaves.

The components of the filtration which contain only an attractor or a repeller are foliated by Reeb components as in [G]. Each such filtration component is 
homeomorphic to a solid torus $S^{1} \times D^{2}$ with the flow oriented in (or out) on the boundary and every orbit entering (or exiting) the component limits on the attractor (or repeller) in forward (or backward) time. The foliation on such a component is sketched in figure 1.

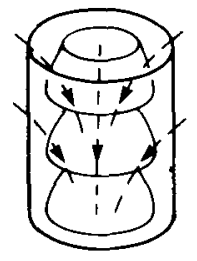

Attractor

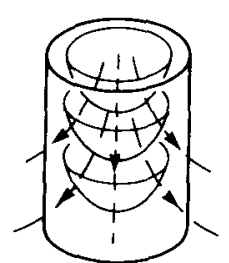

Repeller

FIGURE 1

Next, we will construct a foliation of $\overline{M_{i}-M_{i-1}}$, where $\Lambda_{i}$ is basic set with 2-dimensional unstable manifold. We denote $\overline{M_{i}-M_{i-1}}$ by $X$ and $\partial M_{i}$ by $\partial^{+} X$ (where $\varphi$ is oriented in towards $X$ ), $\partial M_{i-1}$ by $\partial^{-} X$ (where $\varphi$ is oriented out from $X$ ).

Foliation of a neighbourhood of $\Lambda_{i}$. We draw on results of Franks [Fra2] and Birman and Williams [B-W] for the topological structure of $X$, for $\Lambda_{i}$ not an attractor or repeller. There is a neighbourhood $K$ of $\Lambda_{i}$ contained in $X$ which consists of a finite union of blocks as shown in figure 2, where the tops $\left(T_{1}, T_{2}\right)$ are identified with bottoms $\left(B_{1}, B_{2}\right)$ of possibly different blocks. The front, back and bottom arch are contained in $\partial^{-} X$ (where the flow is transverse out); the two sides and the top arch are contained in $\partial^{+} X$ (where the flow is transverse in toward $K$ ). These are bevelled edges joining the pieces of $\partial^{+} X$ to $\partial^{-} X$, to which the flow is tangent. We assume $\varphi$ has a downward component everywhere.

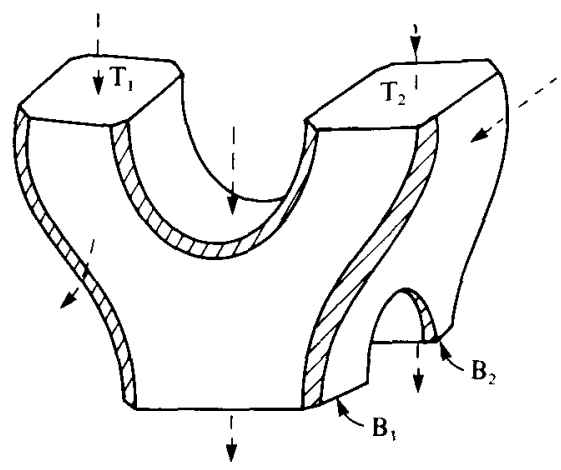

FIGURE 2

Note that in Franks' paper, he requires that $X$ contain codimension-one submanifolds with boundary $U \subseteq V$ tranverse to $\varphi$ such that:

(i) the first return map $r: U \rightarrow$ int $V$ is well defined, smooth and there is a hyperbolic handle set $H \subseteq$ int $U$ with every orbit of $\Lambda_{i}$ intersecting $H$ and every handle $h_{j} \subseteq H$; and 
(ii) if $x \in H$ but $r(x) \notin H$ then $\varphi_{t}(x) \cap H=\varnothing$ for all $t>0$ and the orbit of $x$ meets $\partial^{-} X$ in future time (similarly for $r^{-1}$ ). In dimension 3 these conditions are automatically satisfied; see, for example [Ne] or [B-W].

As Franks shows, $\bigcup_{t} \varphi_{t}(H)$ is doubly foliated by unstable and stable foliations (which correspond to the usual unstable and stable manifolds for points in $\Lambda_{i}$ ). Collapsing along the stable foliations gives the knot-holder of Birman and Williams for $\Lambda_{i}$. We remark that Birman and Williams have shown that the periodic orbits of $\Lambda_{i}$ are in 1-1 correspondence with the periodic orbits of the semi-flow on the knot-holder. On any finite set of periodic orbits, the correspondence is via isotropy in $X$. We will make use of this later.

Each block as in figure 2 is easily foliated transverse to $\varphi$, and transverse to $\partial(X) \cap K$ by horizontal leaves. The induced foliation on the top and bottom arch has an interval in the centre which is tangent to the foliation. We can eliminate the tangencies in the interior of the arch by perturbing the leaves in a small neighbourhood of the interval. If we lift the leaves slightly in the front, the top arch has the induced foliation shown in figure 3(a). If we lift the leaves in back, it has the foliation shown in figure 3(b). A similar modification can be done on the bottom arch. This type of modification to eliminate an interval of tangencies will be used several times in our proof. The decision of which direction to tilt the leaves on any block will be made later.
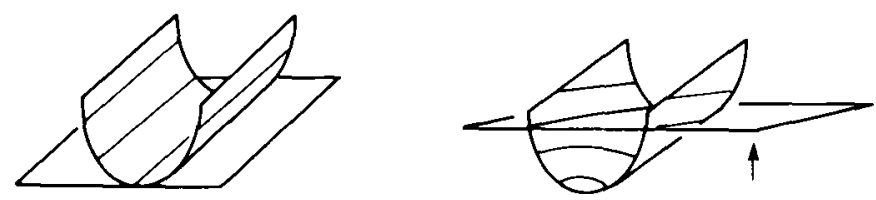

FIGURE 3(a)
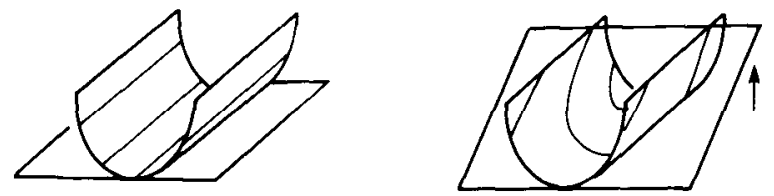

FIGURE 3(b)

Since the foliation is tangent to the tops and bottoms on each block, the various blocks can be easily identified preserving the foliation. We now have a foliation of the neighbourhood $K$ of $\Lambda_{i}$ transverse to $\varphi$. Notice that we have not yet made use of the linking property. This will come next, as we extend the foliation to the rest of $X$, staying transverse to $\varphi$ and to $\partial X$.

Extension of the foliation to $X$. Note that $K \cap \partial^{+} X$ is a 2-manifold with boundary a finite union of circles. It is composed of the inverted $Y$-shaped piece on the sides of a block and the top arches. The edges of the arches and the top edges of the inverted $Y$ 's are identified with the bottom edges of the inverted $Y$ 's. A deformation 
retract of $K \cap \partial^{+} X$ is a 1-dimensional graph with one vertex on each inverted $Y$ (at the branch point) and one vertex in the middle of each arch. The edges of the graph have a natural orientation induced by the flow.

Also note that there is at least one closed oriented loop on this graph. Begin at any vertex and travel back, against the orientation. (In this direction, the path is well-defined.) At some point, since the graph is finite, and since it can never enter an arch for orientation reasons, the path must close up. It may not return to its starting point, but the path contains a closed oriented loop.

We now use the linking property for the first time.

Lемма 3.1. If $\varphi$ satisfies the linking property, $\partial^{+} X$ is a finite union of tori, for all $i$.

Proof. Suppose some component of $\partial^{+} X$, say $N$, is homeomorphic to $S^{2}$. Let $\alpha$ be a closed oriented loop of the graph described above. Then $\alpha$ is a closed curve on $N \cap K$, hence null-homotopic in $N$. The curve $\alpha$ may be isotoped into the interior of $K$ to a periodic orbit of the semi-flow on the knot-holder for $\Lambda_{i}$. Hence by [B-W], it is isotopic to a periodic orbit $\bar{\alpha}$ of $\Lambda_{i}$. Since $\alpha \simeq 0$ on $N, \bar{\alpha}$ is null-homotopic in $X$, contradicting corollary 2.3 .

$M_{i}$ is a manifold with boundary, say $\left(N_{1}=N\right) \cup N_{2} \cup \cdots N_{l}=\partial M_{i}=\partial^{+} X$. It has a non-singular flow $\varphi / M_{i}$, transverse in $\partial^{+} X$. A theorem of Reinhart [R] says that

$$
0=\operatorname{index} \varphi=\frac{1}{2}\left(-\sum_{j=1}^{l} X\left(N_{j}\right)\right) \text {. }
$$

We have just shown that $X\left(N_{j}\right) \leq 0$ for all $j$, so $0=\sum_{j=1}^{l} X\left(N_{j}\right)$ implies $X\left(N_{j}\right)=0$ for all $j$. Therefore every component of $\partial^{+} X$ is homeomorphic to $T^{2}$.

LEMMA 3.2. On each (toral) component $N$ of $\partial^{+} X$, there is a foliation $F$ transverse to the graph retract of $\partial K \cap N$ with the transverse orientation of the foliation corresponding to the orientation on the graph except for a point of tangency at the vertex of each arch, and without Reeb components.

Proof. As we saw in the proof of lemma 3.1, any closed oriented loop on the graph corresponds (under isotopy) to a periodic orbit of the basic set $\Lambda_{i}$ since it isotopes to a knot on the knot-holder for $\Lambda_{i}$. Hence by the linking property, the loop must be essential in $\pi_{1}\left(\partial^{+} X\right)$. Further, no two closed oriented loops intersect since branching of the graph occurs in only one direction. Hence the set of closed oriented loops on the graph restricted to any (toral) component of $\partial M_{i}$ consists of parallel essential curves (with possibly different orientations). The set determines up to sign a generator of the fundamental group of the torus. Take an infinite cycle covering of the torus determined by a homomorphism $\pi_{1}\left(T^{2}\right) \rightarrow \mathbb{Z}$ with value \pm 1 on this generator. Now split each arch at its vertex and let each half go off to that same end of the cylinder that the $Y$-shaped piece it is attached to does.

The orientation of the graph identifies a positive end of each component of the graph, which corresponds to one end of the cylinder, and a negative end of each component which corresponds to the other end of the cylinder. Of course, two components may switch positive and negative ends. 
If it is the case that all the components have the same positive end, one may approximate the graph with a flow, which by [Fri] has a cross-section.

So the graph on the cylinder is transverse to a foliation of the cylinder by circles. We may then rejoin the arches that were split in the step above so that each arch is transverse to the foliation except for a single tangency at the vertex. Now project back to the torus. There we have the desired foliation $F$. See figure 4(a) for an illustration of this procedure.
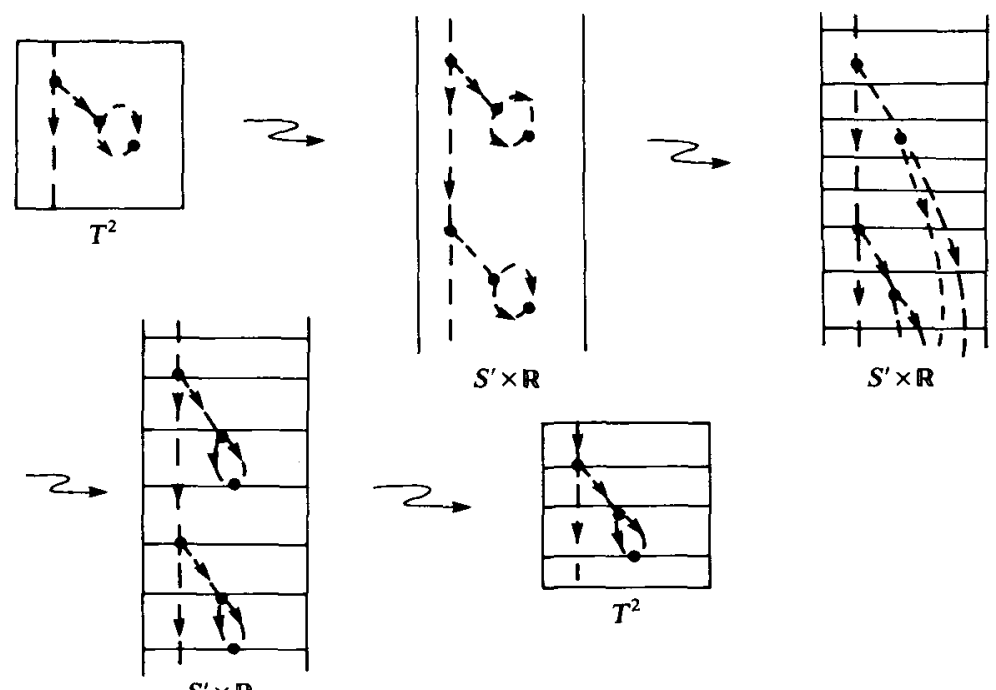

$S^{\prime} \times \mathbf{R}$

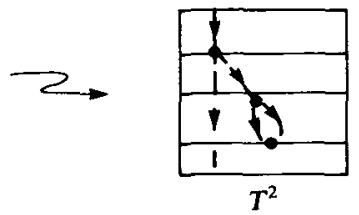

Figure 4(a)
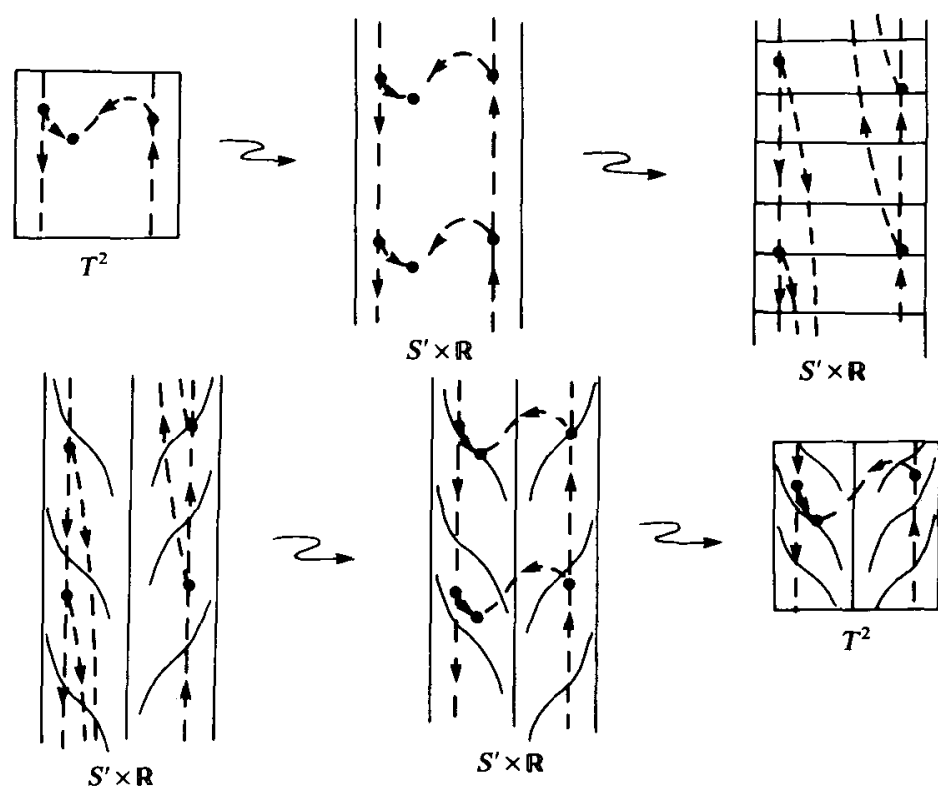

Figure 4(b) 
Now suppose that the components do not all have the same positive and negative ends. If the orientation were reversed on the components which have the opposite positive end from a given one, the lifted and split graph would be transverse to a foliation by circles once again. We will alter this foliation to change the transverse orientation where needed. Separate adjacent components of the graph with opposite positive ends by a copy of the real line, not intersecting the graph, going from one end of the cylinder to the other and intersecting the circle foliation transversely. Now 'turbularize' ([No]) the foliation near the line (away from the graph) so that the line becomes a leaf, the circles split to approach that leaf asymptotically, and the transverse orientation of the foliation is reversed on one side. Then rejoin the arches where they were split so that they are transverse except at the vertices and project to the torus. See figure $4(b)$ for an illustration.

Note that we choose the direction of turbularization to avoid (two-dimensinal) Reeb components. This last condition of no Reeb components will become important later.

Recall that $\partial K \cup \partial^{+} X$ is a 2-manifold (with boundary) consisting of the $Y$ 's from the sides of each block and the top arches. The foliation induced on these pieces by the foliation on $K$ can be made to match the foliation $F$ on $\partial^{+} X$ just constructed by an appropriate tilt of the top arch as referred to in figure 3 earlier. The foliation on the sides clearly matches. The remaining portion of $\partial^{+} X$, that is $\partial^{+} X-\partial K$, is a union of disks and annuli parallel to the circles. Every orbit which enters $\partial^{+} X-\partial K$ exits $\partial^{-} K-\partial K$ without entering $K$. Hence there is a homeomorphism induced by the flow $\varphi$ between $\partial^{+} X-\partial K$ and $\partial^{-} X-\partial K$. So $X-K$ consists of pieces homeomorphic to $N \times[0,1]$, where $N$ is a disk or annulus and where the orbits of $\varphi$ on each piece may be assumed to be $\{x\} \times[0,1], x \in N$. We will use $F$ on $N \times 0 \subseteq \partial^{+} X$ to extend the foliation on $K$ to all of $X$.

Let $N$ be a disk or annulus in $\partial^{+} X-\partial K$. There is a foliation induced on $N$ by $F$, with an even (possibly zero) number of tangencies of $\partial N$. Foliate $N \times[0,1]$ by the product foliation $\bar{F}$ given by the $F / N \times[0,1]$. $\bar{F}$ on $N \times[0,1]$ is tangent to $\varphi$ and has intervals of tangencies corresponding to the tangencies on $\partial N$. By perturbing $\bar{F}$, leaving it fixed on $\partial^{+} X$, we will make it transverse to $\varphi$ and match the foliation on $\partial N \times[0,1]$ induced by $K$, which we shall denote by $F_{K}$.

First of all, notice that $F_{K}$ on $\partial N \times[0,1]$, single annulus or two, has an even (possibly zero) number of 'Reeb panels', one for each tangency on $\partial N \times\{0\}$. For orientation reasons, these panels alternate in direction. See figure 5 for an illustration.

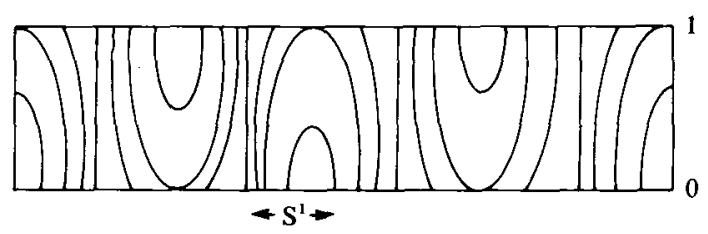

FIGURE 5 
Notice also that the product foliation $\bar{F}$ on $\partial N \times[0,1]$ has, again for orientation reasons, intervals of tangencies which alternate between outer tangencies (like the two local maximums in figure 6) and inner tangencies. On $\bar{F}$, there is a transverse orientation induced by a transverse orientation on $F$. Tilt the leaves of $\bar{F}$ back with respect to this transverse orientation as they go from $\partial^{+} X$ to $\partial^{-} X$. This not only makes the foliation on $N \times[0,1]$ transverse to $\varphi$; it makes the foliation on $\partial N \times[0,1]$ match $F_{K}$. (See figure 3 again.) Two examples of this perturbation are shown in figure 6 .
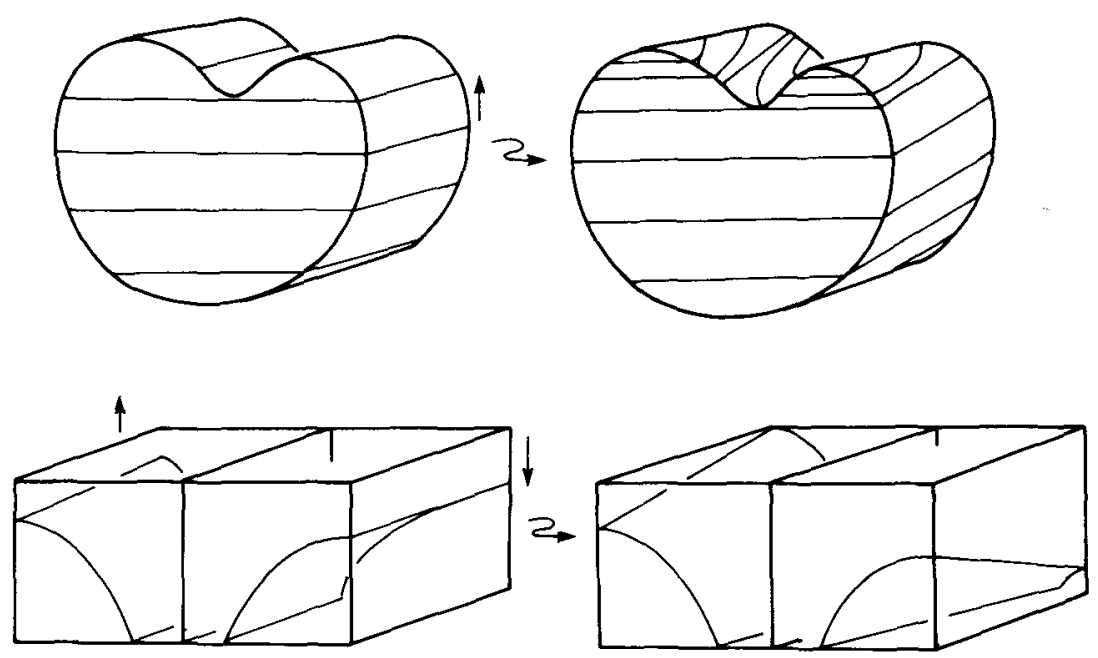

FIGURE 6

In spite of the care taken in lemma 3.2 to produce a foliation of $\partial^{+} X$ with no two-dimensional Reeb components it may happen that the extended foliation of $\partial^{-} X$ has Reeb components (in the case of twisted connectors). In the next section, the foliation is spun near $\partial X$ to make the boundary components leaves of the foliation. Hence we must eliminate these Reeb components on $\partial^{-} X$. This may be done as follows:

Let $R\left(\cong S^{1} \times[0,1]\right)$ be a two-dimensional Reeb component on $\partial^{-} X$. Let $U \cong$ $S^{1} \times[-\varepsilon, 1+\varepsilon]$ be a neighbourhood of $R$ in $\partial^{-} X$. Let $U \times[0, \varepsilon]$ be a one-sided collar of $U$ in $\bar{M}_{i-1}$ such that the flow $\varphi$ is transverse to $U \times\{t\}, t \in[0, \varepsilon]$. Foliate $U \times[0, \varepsilon]$ transversely to $\varphi$ in the following way. There is a leaf $L$, homeomorphic to $S^{1} \times[0,1]$ with $\partial L=S^{1} \times\{0,1\}=\partial R \subseteq \partial^{-} X$. The leaves in the interior of $R$ extend to approach $L$ asymptotically. On $\partial U \times[0, \varepsilon] \cup U \times\{\varepsilon\}$, the foliation is trivial with leaves being level sets in the $S^{1}$ direction. These leaves also extend to the interior approaching $L$ asymptotically. See figure 7 for an illustration of the foliation of $U \times[0, \varepsilon]$. 


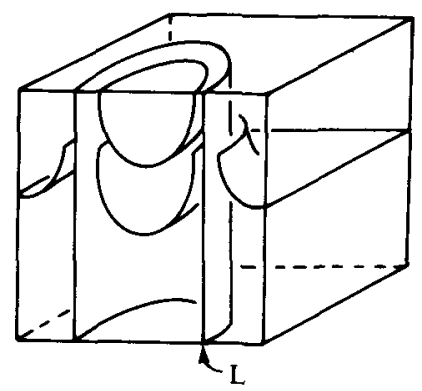

Figure 7

Now extend $M_{1}$ slightly to include $U \times[0, \varepsilon]$. We have reduced the number of Reeb components on $M_{i-1}$ by at least one. Repeat this process until all Reeb components on $M_{i-1}$ are eliminated.

Turbularization near the boundary. We now have a foliation of $X=\overline{M_{i}-M_{i-1}}$ transverse to $\varphi$ and transverse to $\partial X$. The foliation restricted to any component of $\partial X$ is either a trivial foliation by circles or a foliation with some compact circles leaves, and the other leaves noncompact, approaching these circles asymptotically (but without Reeb components). In either case the foliation may be modified in a small collar neighbourhood of the boundary, sweeping the leaves asymptotically around the toral boundary components, and making the boundary tangent to the foliation (called turbularization in [No]). Since $\varphi$ is tangent to the boundary, this may be done keeping the foliation transverse to $\varphi$.

We now have a foliation on $M_{0}$ and $\overline{M_{i}-M_{i-1}}$ for each $i$, transverse to $\varphi$. Since the foliations are all tangent to the boundary, they may clearly be glued together along the boundary tori to produce a foliation of all $M$ transverse to $\varphi$. This completes the proof of sufficiency of the linking property for flows with a hyperbolic 1-dimensional chain recurrent set.

COROLlaRY 3.3. For a non-singular vector field $\varphi$ with a 1-dimensional hyperbolic chain recurrent set, the following are equivalent:

(i) $\varphi$ satisfies the linking property;

(ii) no periodic orbit in a basic set $\Lambda_{i}$ is null-homotopic in $\overline{M_{i}-M_{i-1}}$.

(iii) there is a 2-dimensional foliation everywhere transverse to $\varphi$.

Remark. In any component where the foliation on $\partial M_{i}$ is not a trivial foliation by circles, the foliation after turbularization is only $C^{1}$-smooth at the boundary torus. See ([G]) for a proof. As we saw there, some flows which have transverse foliations do not have $C^{2}$-smooth ones.

Remark. If the foliation $F$ (from lemma 3.2) is a trivial foliation by circles for each component of $\partial M_{i}$, the flow $\varphi$ restricted to $\overline{M_{i}-M_{i-1}}$ has a cross-section (transverse to $\partial M_{i} \cup \partial M_{i-1}$ ), This is a straightforward application of Fried's ([Fri]) or Schwartzman's ([Sch]) work. So in that case $\overline{M_{i}-M_{i-1}}$ fibres over $S^{1}$. But this is certainly not true in general. In fact, we see homology directions which are antipodal. 
Remark. The flow having a transverse foliation seems to place no restriction on the ambient manifold or the homotopy class of the flow (assuming Smale flows are $C^{0}$-dense). If $\varphi$ is any non-singular flow on any 3-manifold, it may be homotoped to a flow $\psi$ with a transverse foliation, since any 2-plane field may be homotoped to an integrable one ([T]). Then $\psi$ may be approximated by a Smale flow $\psi^{\prime}$ which is still tranverse to the foliation.

\section{REFERENCES}

[A-G] D. Asimov \& S. Goodman. Stable non-singular flows not transverse to any foliation. Preprint, 1978.

[B-W] J. Birman \& R. F. Williams. Knotted periodic orbits in dynamical systems II: Knot holders for fibred knots. In Low Dimensional Topology. Amer. Math. Soc. (1983), 1-60.

[B] R. Bowen. One-dimensional hyperbolic sets for flows. J. Diff. Eq. 12 (1972), 173-79.

[E-H-N] D. Eisenbud, U. Hirsch \& W. Neumann. Transverse foliations of Seifert bundles and selfhomeomorphisms of the circle. Comm. Math. Helv. 56 (1981), 638-660.

[Fra1] J. Franks. Homology and Dynamical Systems. CBMS Regional Conf. Series in Math. 49, Amer. Math. Soc.

[Fra2] J. Franks. Non-singular Smale flow on $S^{3}$. Topology 24 (1985), 265-282.

[Fri] D. Fried. Cross-sections to flows. Topology 21 (1982), 353-372.

[G] S. Goodman. Vector fields with transverse foliations. Topology 24 (1985), 333-340.

[M] J. Morgan. Non-singular Morse-Smale flows on 3-dimensional manifolds. Topology 18 (1978), 41-53.

[Ne] S. Newhouse. The abundance of wild hyperbolic sets and non-smooth stable sets for diffeomorphisms. Publ. I.H.E.S. 50 (1979), 101-152.

[No] S. P. Novikov. Topology of foliations. Trans. Moscow Math. Soc. 14 (1965), 268-304.

[O] M. Oliveira. $C^{0}$-density of structurally stable dynamical systems. Thesis, Univ. of Warwick, 1976 (revised 1979).

[R] B. Reinhart. Cobordism and the Euler number. Topology 2 (1963), 173-177.

[Sch] S. Schwartzman. Asymptotic cycles. Annals of Math. 66 (1957), 270-283.

[Sm] S. Smale. Differentiable dynamical systems, Bull. Amer. Math. Soc. 73 (1967), 747-817.

[Th] W. Thurston. A local construction of foliations for 3-manifolds. In Amer. Math. Soc. Proc. Symp. Pure Math. 27 (1975), 315-319.

[W] J. Wood. Bundles with totally disconnected structure group. Comm. Math. Helv. 46 (1971), 257-279.

[Z] E. C. Zeeman. Morse inequalities for diffeomorphisms with shoes and flows with solenoids. In Dynamical Systems - Warwick 1974. Lecture Notes in Maths. 468, Springer (1975), 44-48. 